\title{
Balancing Individual Capabilities and Social Peer Pressure for Role Adoption
}

\author{
Xin Zhang ${ }^{1}$ and Henry Hexmoor ${ }^{2}$ \\ ${ }^{1}$ Department of Computer Science \& Engineering, \\ Arizona State University, Tempe, AZ 85287, USA \\ xin.zhang@asu.edu \\ 2 Department of Computer Science \& Computer Engineering, \\ University of Arkansas, Fayetteville, AR 72701, USA \\ hexmoor@uark.edu
}

\begin{abstract}
In this paper, we model agent role adoption when they are under the influence of peers and must account for their capabilities as well. Our model suggests a strategy for role adoption that decreases tension among agents. We show the contention between considering social pressure versus role qualification as two factors for role adoption.
\end{abstract}

\section{Introduction}

In multiagent systems, roles have been modeled in planning and distribution of tasks [4][5][9][21]. This work has focused on norms, obligations, rights and other interpersonal protocols that govern role adoption. Roles are sometimes terminal goals. At other times, adoption of a role is itself an achievement goal. Often, satisfaction of a goal propels the agent to adopt a role. But in some cases, an agent adopts roles not based on goals, but on norms, obligations, and values [6][7]. Little attention is paid to an agent's preferences over reasoning about role adoption under social influences, which is a kind of peer pressure. In a society, since agents who possess roles have social interactions with one another, a role is more than a task [3]. One way that roles are different than tasks is that individuals tend to have preferences for these roles. An individual's perceptions and preferences about roles are shaped by their social kin and peers. In this paper, ${ }^{1}$ we will consider individual role adoption when the agent considers peer pressure as influences it perceives in its social network.

Social network analysis (SNA) focuses on studying the behavior of an individual at the micro level, the pattern of relationships at the macro level, and the interactions between the two [20]. Social structures capture the patterns of connections and interdependencies among organization members [24]. When an organization is viewed formally, connections in a social structure may reflect authority relationships, whereas in an informal view of an organization, communications reflect social relationships [24]. The interaction patterns describing social structures can be viewed as a network of relationships [18]. The network paradigm is an ideal way to examine

\footnotetext{
${ }^{1}$ This work is supported by AFOSR grant F49620-00-1-0302.
} 
a social organization based on the view of actors as an interconnected set of interdependent relationships instead of independent actors [8]. An individual's pattern of relations is called a role set, and people playing a similar role are said to occupy similar structural or status positions [24]. French's theory [12], formulated a model of how people's opinions are affected by the opinion of other persons with whom they are in direct communication. At time $t$, all people move to positions that minimize the sum of the squared distances among them and other influential opinions at time $t-1$. In Friedkin's model [13], members of an influence structure change opinions based on the value of their own opinion and the opinion of those members in the influence structure affecting them. Three factors determine dyadic agreement: path length, path redundancy, and path centralization. This predicts that the longer the path connecting nodes, the smaller the path's contribution to the probability of dyadic agreement. The greater the number of paths of a particular length connecting two nodes, the more likely the two nodes will be in agreement. Fritz Heider developed balance theory [14] in the area of social perception and attitudes. The theory posits that the mind seeks balance (an absence of tension) by trying to hold ideas that are not in conflict with one another. This theory also applies to attitudes towards other people. He was especially concerned about what happens when a person is emotionally close to two people who start becoming hostile to each other. For example, assume that we have three agents $A, B$ and $C$, and two kinds of relationship like and dislike. If $A$ likes $B$, then $A$ wants to like and dislike all the things that $B$ likes and dislikes. If $B$ dislikes $C$, then $A$ wants to dislike $C$. But if $A$ and $C$ have strong positive relationship, there is a tension that have to be solved. In the next section we will show how we apply the above relationship in our model of role adoption. The basic balance theory hypothesis is that people who find themselves in an unbalanced position should change their relations to generate balance [19]. Hypothetically, this process will lead to greater balance over time.

In sociology, role theory goes back to 1950s. With the development of multi-agent systems that simulated human behaviors by artificial agents the concept of role has undergone renaissance [22]. Currently, the research in agent role adoption has been focused on agent's current roles [15]. Roles cannot be independent of the sociological structure or the environment where they evolve. Social Role Valorization (SRV) Theory is formulated in 1983 by Wolf Wolfensberger as the successor to his earlier formulation of the principle of normalization [23]. The major goal of SRV is to create or support socially valued roles for people within their society, since if a person adopts valued social roles, the person will be highly likely rewarded from that society. In other words, all sorts of good things that other people are able to convey are almost automatically accorded to a person who holds socially valued roles, at least within the resources and norms of the society [16].

In this research, we consider role adoption based on influences of peer agents who are in the agent's social network. Role adoption based on social network consideration can be modeled as the function of a local view and a global view of role adoption with social connections. When viewed locally, agent role adoption in social network will be based on the balance theory and centralization to reduce the tension for each individual agent. For the global view of role adoption, which is not explored further in this paper, it will be based on a theory such as the Social Role Valorization Theory such that if the agent adopts the roles preferable by the society, it will be awarded to have higher motivation to be active in social network. 
This paper is structured as follows. In section 2, we describe a model of the role adoption based on social network, where we include a number of definitions. In section 3, we describe our implementation and the experiments we conducted that show combinations of social influence and capability for role adoptions. Finally, section 4 offers concluding remarks and future works.

\section{A Model of Role Adoption under Influence of Peer Network}

A social network can represent relationships between agents. Agent social networks can be used to model peer influences and increase homogeneity among used agent's role adoption. It has been found that same-cluster members tend to be more similar in their opinions than different-cluster members [1]. A cluster can be viewed as an organization. Similarly, according to balance theory when agents adopt roles based on preference, they are likely to adopt the same type of roles that have been adopted by the agents with whom they have strong relationship and mutual influence in order to reduce tension among them. A dominant concept in social network analysis is centrality [10][11]. Actors are more central to the extent that they have more relationships with more members of the network, i.e., play a role that is more connected to other roles [24].

We focus on a dyadic relationship in a network with the properties of symmetry and transitivity [17]. Social networks are commonly represented by a graph. Each agent in the social network is a vertex, and the social relationship between two agents is an edge. Social relationships between agents have different potency among agents. If the relationship between agents is strong, the value will be large. We can use an $n$ by $n$ matrix whose elements are valued between 0.0 to 1.0 to represent relationships in agent social network graphs, where $n$ is the number of agents. The relationship we are exploring is peer pressure for role adoption [2]. Agents with larger relationship values exert larger pressure on one another to adopt the same type of role. We realize peer pressure is only one of a myriad of influences individuals may have on one another. For example, a parent may influence a child about a role but this relationship is typically not symmetric. Henceforth, we will refer to a network of peer pressure values among agents as peer network. In this paper, handling of cycles in the network relationship is not covered.

Definition 1 Social peer pressure value (we abbreviate with peer value in the rest of this paper) is the strength of the peer pressure value between two agents.

The relationship can be direct relationship as well as indirect relationship, which means that the agents have strength of the peer value via intermediary agents. Two agents can be connected via different routes with the same length. We define the concept of route set to collect all the routes connecting two agents with the same length.

Definition 2 Route set, $R_{i}(a, b)$, is the aggregation of all routes connecting agents $a$ and $b \quad$ with $\quad$ length $i . \quad$ I.e.,

$R_{i}(a, b)=\{r \mid r$ is a path (a succession edges) of length $i$ between agent $a$ and $b\}$ 
If two agents are not directly connected, the distance (i.e., the number of edges) between two agents will affect the strength of pressure. We define the influence value to represent the peer pressure between two agents via certain routes.

Definition 3 Peer Influence value, Value $e_{e_{1}, e_{2}, \ldots, e_{n}}(a, b)$, is the peer pressure value between agents $a$ and $b$ via route $e_{1}, e_{2}, \ldots, e_{n}$, which connects agents $a$ and $b$. I.e., $a, e_{1}, e_{2}, \ldots, e_{n}, b \in R_{n+1}(a, b)$, where $e_{i}$ is an edge.

(1) if agents $a$ and $b$ are directly connected, the value is defined in social peer network matrix;

(2) If agents $a$ and $b$ are not directly connected, such that is connected via route $e_{1}, e_{2}, \ldots, e_{n}$,

$$
\operatorname{Value}_{e_{1}, e_{2}, \ldots, e_{n}}(a, b)=\operatorname{Value}\left(a, e_{1}\right) \cdot\left(\prod_{i=1}^{n-1} \operatorname{Value}\left(e_{i}, e_{i+1}\right)\right) \cdot \operatorname{Value}\left(e_{n}, b\right) ;
$$

where $\operatorname{Value}\left(a, e_{1}\right)$,Value $\left(e_{i}, e_{i+1}\right)$ and $\operatorname{Value}\left(e_{n}, b\right)$ is defined in the social peer network matrix. For any agent a, $\operatorname{Value}_{\varnothing}(a, a)=1$.

Agents influence one another following certain routes. For any two agents $a$ and $b$, they may influence with one another at levels of the different lengths of the route.

Definition 4 Route Set Value between agents $a$ and $b$ via $R_{i}(a, b)$, denoted by RouteSetValue $_{i}(a, b)$ is

$$
\text { RouteSetValue }_{i}(a, b)=\max \left[\sum_{\text {for all }} \text { Value }_{\mathrm{r}_{i}}(a, b), 1\right]
$$

Route Set Value is reciprocal, i.e., agent $a$ 's capability to influence is the same as agent $b$ 's capability to influence. The influences between any two agents are the effects of the influences from all lengths of the routes.

Definition 5 Total Value between agents $a$ and $b$, denoted by $\operatorname{TotalValue}(a, b)$ is:

$$
\operatorname{TotalValue}(a, b)=\sum_{i=1}^{i=d} W_{i} \bullet \operatorname{RouteSetValue}_{i}(a, b)
$$

where $d$ is the distance between agents and $W_{i}$ is the relative weight for influence, which satisfies $W_{1}+W_{2}+\cdots+W_{d}=1, W_{1}>>W_{2}>>\cdots>W_{d}$.

Intuitively, the nearer the distance, the more influence the agent has. The reason that we only calculate up to distance $d$ instead of the largest distance between the agents is that we assume that if the connection distance is too long, the influence is negligible. For practical reasons, we suggest keeping $d$ under 3.

Definition 6 The Average Pressure for an agent in a social network for agent $a$, denoted by Average Pressure $(a)$, is the average of influence value of agent $a$ with its neighbors with whom it has direct connections.

$$
\text { Average Pressure }(a)=\sum_{\text {for All agent b }} \operatorname{TotalValue}(a, b) / \text { num_connection }
$$

where num_connection is the number of direct connects that agent $a$ has. 
Agents consider peer network influence based on both average pressure from the connections and the number of connections.

Definition 7 Social network peer value, denoted by NetworkPeerValue( $a$ ), is the weighted sum of number of connections and the average pressure of connections. I.e.,

$\operatorname{NetworkPeerValue}(a)=w_{1} \cdot$ Average Pressure $(a)+w_{2} \cdot$ num_connection (6)

where $w_{1}$ and $w_{2}$ are relative weights and $w_{1}+w_{2}=1.0$.

If two agents have the same strength of connection, the agent who has more connections has more influence and vice versa. If we assume that we have a total of $M$ agents and $N$ roles, each agent has a role and each role requires $m$ abilities. We expect each agent to adopt one and only one of the roles, and a role can be adopted by any number of agents. Besides, the agents have different pressure relationship in the peer network. They also have different capabilities, say agent $A_{i}$ has $m$ abilities, denoted by $C_{A_{i}}^{1}, \ldots, C_{A_{i}}^{m}$, where $0 \leq C_{A_{i}}^{j} \leq 1,1 \leq j \leq m$. Whereas roles require various capabilities, say role $R_{j}$ requires $m$ abilities, denoted by $C_{R_{j}}^{1}, \ldots, C_{R_{j}}^{m}$ for the agents to perform, where $0 \leq C_{R_{j}}^{k} \leq 1,1 \leq k \leq m$. When an agent adopts a role without considering social network, the agent will find the role that best matches its capabilities. Intuitively, the agent will take the roles that it fits best.

Definition 8 The best match value, denoted by BestMatchValue, for agent $i$ is as follows:

$$
\text { BestMatchValue }(i)=\max \left[\sum_{k=1}^{m}\left(\frac{C_{A_{i}}^{k}}{C_{R_{1}}^{k}}\right), \ldots, \sum_{k=1}^{m}\left(\frac{C_{A_{i}}^{k}}{C_{R_{N}}^{k}}\right)\right]
$$

where $m$ is the total number of capabilities that the agents have and the role requires. $N$ is the number of roles.

Each agent will compare its abilities with each role, and find the best role it fits. However, more realistically influences of peer network on agent's role adoption should also be considered. When an agent chooses the same role as the agents that have strong social connection with it, the agent can exchange more experience with others, gain more utility, and have higher motivation to perform the role. Therefore, the strategy of role adoption is based on both agent capability match and the social peer network influence.

Definition 9 The role utility for agent $i$ is the weighted sum of capability match and social network influence:

RoleUtility $(i)=W_{1} \cdot$ BestMatchValue $(i)+W_{2} \cdot$ NetworkPeerValue $(i)$

where BestMatchValue(i) is the role adoption value that the agent $i$ will adopt the role that best matches its capability; here, . $W_{1}+W_{2}=1.0$.

Here we assume all agents have the same weights for best match versus influence. This will help us isolate the effects of these weights in a community of agents who share a similar weight. This is a simplifying assumption in our model. Generally, individuals will have different weights.

When performing a role long enough, the agent will exchange the experience with some other agents who perform the same type of role. So agents will get experience gain after performing the roles.

Definition 10 Experience gain, denoted by ExperienceGain(a), for agent $a$ is: 


$$
\text { ExperienceGain }(a)=W_{1} \cdot t+W_{2} \cdot \sum_{\text {for all agent i }} \operatorname{TotalValue}(a, i)
$$

where $t$ is the duration for performing the role, $W_{1}$ and $W_{2}$ are relative weights and $W_{1}+W_{2}=1.0$.

Definition 11 Intra Role Tension between a pair of agents $a_{i}$ and $a_{j}$, denoted by IntraRoleTension $\left(a_{i}, a_{j}\right)$, is defined as follows:

a) IntraRoleTension $\left(a_{i}, a_{j}\right)=1$-Influence_value $\left(a_{i}, a_{j}\right)$, if $a_{i}$ and $a_{j}$ adopt same type of roles;

b) IntraRoleTension $\left(a_{i}, a_{j}\right)=0$, otherwise, i.e., if $a_{i}, a_{j}$ have different roles.

Whereas influence value is the amount of pressure and agreement two agents have about a role, intra role tension is the compliment of this value and reflects the disagreement between them. When influence is at maximum, this value is at 1.0 and there is no disagreement. Values below 1.0 leave some room for disagreement.

Definition 12 Intra Role Tension for a group of agents $A=\left\{a_{i} \mid a_{i} \in A\right\}$, denoted by $\operatorname{RoleConflict}(A)$, with the same type of role is defined as follows:

$$
\operatorname{RoleConflict}(A)=\sum_{i=1}^{i=n} \sum_{j=1}^{j=n} \operatorname{IntraRoleTension}\left(a_{i}, a_{j}\right) \text {, }
$$

where $\mathrm{n}$ is the size of group of agents $\mathrm{A}, a_{i}, a_{j} \in A, i \neq j$

Definition 13 Role Satisfaction for an agent $a$, denoted by RoleSatisfaction( $a$ ), is the difference between the sum of the influence value of the same type of relationship and the sum of the influence value of the different type of relationships, divided by the number of the relationships.

$$
\operatorname{RoleSatisfaction}(a)=\left(\sum_{b_{i} \in B} \operatorname{NetworkPeerValue}\left(a, b_{i}\right)-\sum_{c_{i} \in C} \operatorname{NetworkPearValue}\left(a, c_{i}\right)\right) / n
$$

where B is the group of agents that adopt the same type of roles as agent a, $\mathrm{C}$ is the group of agent that adopt the different type of roles of agent a. " $n$ " is the number of agent $a$ 's relationships.

\section{Experiments}

We designed and implemented a system that simulated a town in an isolated island. We considered three roles of Doctor, Rancher, and Farmer, which we will refer to them as R1, R2, and R3. Let's assume three general capabilities C1, C2, and C3. We used a role capability table (not shown) to specify how much (a number between 0.0 and 1.0) capability is required for each of the three roles. Each agent is assumed to have a static capability. We used an agent capability table (not shown) to specify these static values. There may not be a perfect match between agents and roles. However, agents match roles to differing degrees. The simplest role adoption strategy for an agent is based on best match between its capability and needed roles. When 
considering social influences, agents will change roles they adopt based on both social pressures and their matching ability. The social network strength of influence between agents is randomly generated among agents. The following is the basic control loop for each agent:

\section{Initialize Role-Capability, Agent-Capability tables \\ 2. Loop \\ a. Sense global knowledge \\ b. adopt role \\ c. Perform role \\ d. Consider changing environmental factors that affect role adoption}

Intra Role Tensions between agents is the first parameter we measured. As we defined earlier (see definition 11), intra role tension is a dissimilarity measure (i.e., disagreement) among a group of agents. When agents adopt the same type of roles, we consider them as a group. For each agent in this group, we measure the influence between this agent with all the other agents pair-wise in the group. If there are influences between two agents, we measured the intra role tension as 1.0 minus the current influence. For example, if the agent shares no influence with other agents but adopts the same type of role, the pair-wise intra role tension is 1.0. If two agents have strong agreement such as 1.0 , when they adopt the same type of role, the intra role tension between them is 0.0 . We consider all pair-wise agents to calculate the intra role tensions, and sum them together to get group intra role tensions.

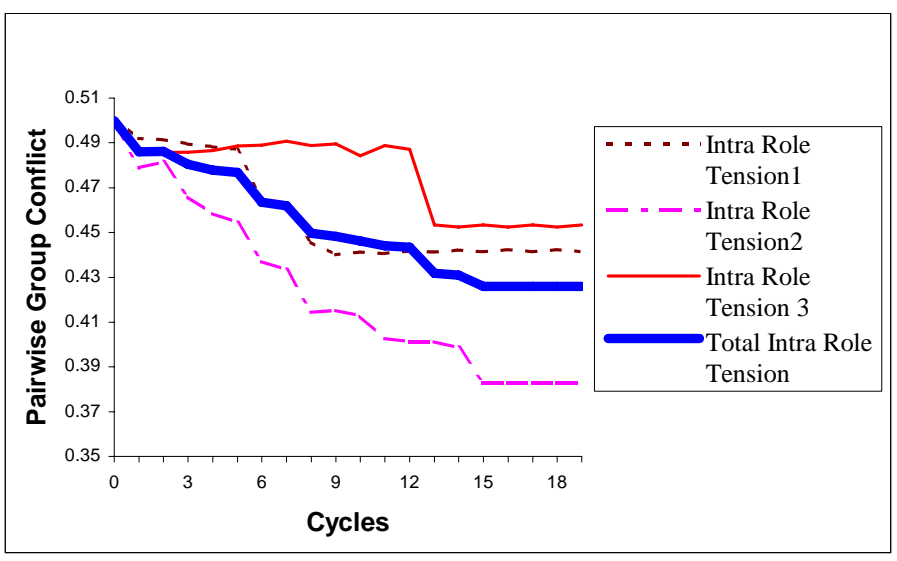

Fig. 1. Intra role tensions with considering Social Network "Cycles” are simulation cycles.

We assigned a weight for considering social network to balance role capability match and peer influence. The results shown as Figure 1 indicate that the intra role 
tension has been steadily decreased when considering the influence of social network. Without considering social network influence, the intra role tension remains unchanged. Within each simulation cycle, each agent considers role adoption with influences from its social peer network. Therefore, in each cycle the number of role considerations is the same as the number of agents. For example, in a simulation with 10 agents, each simulation cycle will have nine considerations for an agent, each for an individual agent.

In the above experiment, we assigned the weight for considering social network at 0.33 , while we assigned the weight of considering ability matching is 0.67 . We found out that when the number of agents increases in our experiment, the effect of peer influence network become stronger. If we maintain the same weights for considering social network influences, say 0.33 and 0.67 , all of the agents are very likely to play the same type of roles, and the intra role tension for the agents will be high. When the number of agents increases (i.e., a larger population), the weight assignment for social network should be adjusted to lower tension. In our experiments, we explored the weights assignments in order to minimize the intra role tensions. We determined what weighting values in equation in definition 9 minimized intra role tension. There is an optimal set of weights that minimize the intra role tensions within the system. Figure 2 shows the relationship between the optimal weight for considering peer network in role adoption and the number of agents.

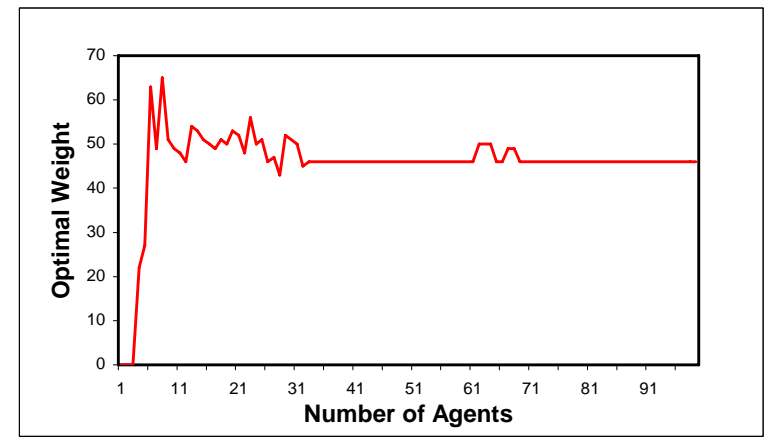

Fig 2. Optimal Weight for Considering Peer Network Role capability match weight is the compliment of the values in the vertical axis.

An agent role satisfaction over time is another quality we measured. As we defined earlier, role satisfaction is the similarity of the agents in peer influence networks. We considered the influences in the social network for each agent. For each agent, the influence between itself and another agent who adopts the same type of role is considered as a positive influence. The influence between the agent itself and an agent who adopts a different type of roles is considered as disagreement and therefore a negative influence. Satisfaction for each agent is then the sum of its positive influences and negative influences divided by the number of agents with whom the agent has been compared to (see definition 13). With considering peer influence, the agents with strong relationship will adopt the similar roles. Also, agents will be more and more satisfied with their roles. Figure 3 shows that the satisfactions among the agents with considering peer influence network are steadily increased in the first 16 | cycles and keep constant after that. 


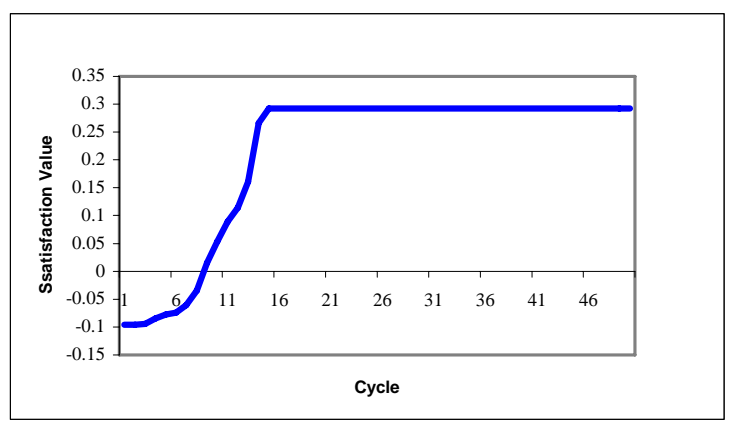

Fig 3. Satisfaction among agents with considering peer network

\section{Conclusion}

We presented a model of agent role adoption when an agent must consider social influences form its peers and its own capabilities. In this work when agents adopt roles within a peer network, they reason based on both attributes of agents' own ability and local social network influences. We discussed the implementation of the model, and analyzed the effect of peer network towards intra role tension, optimal weight for considering social influences, and agent satisfaction towards roles. The results show that by considering social influences of peers, an agent can decrease intra role tension. Also agent satisfaction is increased. The optimal weight for considering social influences is quite constant in communities with a large population, with the value of about 0.48 . The optimal weight for considering peer pressure varies a lot and tends to be small in relatively small populations.

In global view of agent role adoption, agents adopt roles not only based on individual connection and relationships, but also based on the global evaluations by the society. In global point of view, some roles are needed by the society but may not be highly recommended based on agent's ability and agent's individual social relationship. In this case, some agents will sacrifice their individual choice but follow the society needs, socially valued roles. Social Role Valorization Theory assumes that a society will evaluate the roles from global point of view. If agents adopt the socially valued roles, they will be rewarded by the society. In this paper, we focused on the role adoption in the local view. Our future work will combine both views in one framework.

\section{References}

1. Alba, Richard D, Taking stock of network analysis: A decade's result. Perspectives in Organizational Research, Greenwich, CT, JAI press, (ed. S. Bacharach) 1981, 39-74. 
2. Bandura, A. (2001a). Social cognitive theory: An agentic perspective. In S. T. Fiske (Ed.), Annual review of psychology (Vol. 52, pp. 1-26). Palo Alto: Annual Reviews, Inc.

3. Cabri, G. (2001). Role-based Infrastructures for Agents. In Proceedings of the $8^{\text {th }}$ IEEE Workshop on Future Trends of Distributed Computing Systems (FTDCS 2001), Bologna

4. Cavedon, L., and Sonenberg, L., On Social Commitments, Roles, and Preferred Goals, In Proceedings of $3^{\text {rd }}$ International Confernce on Multiagent Systems, pp. 80-87, (1998).

5. Castelfranchi, C. and Falcone, R., From Task Delegation to Role Delegation, In Proedings of AI-IA 1997: Advances in Artificial Intelligence Congress, LNAI 1321, pp. 278-289.

6. Castelfranchi, C., Dignum, F., Jonker, C. M., \& Treur, J. (1999). Deliberate Normative Agents: Principles and Architecture. In N. Jennings and Y. Lesperance (eds.) Proceedings of ATAL-99 , 206- 220.

7. Ch’ng, S., \& Padgham, L. (1997). From Roles to Teamwork: a framework and architecture. Journal of Applied Artificial Intelligence, Vol. 12, special issue on Robocup, 211-231.

8. Contractor, N.S., and Eisenberg, E. M., Communication networks and new media organizations, Chapter 7 in Organizations and Communications Technology, Janet Fulk, Charles Steinfield (eds.), Sage Publications, Newbury Park, 1990, pp. 143-171.

9. Fasli., M. (2001). On Commitments, Roles, and Obligations. $2^{\text {nd }}$ International workshop on Central and Eastern Europe on Multi-agent Systems (CEEMAS 2001), 93-102.

10.Freeman, L.C., Centrality in Social Networks: Conceptual Clarification, Social Networks, No.1, 1979, pp. 215-239.

11.Freeman, L.C., Roeder, D., Mulholland, R. R., Centrality in Social Network: II Experimental Results, Social Networks, Vol.2, 1980, pp 119-141.

12.French, John R.P.Jr, A formal theory of social power. The Psychological Review 63, 1956.

13.Friedkin, Noah E., A formal theory of social power. In Journal of Mathematical Sociology, 1986, Vol. 12(2) pp. 103-126.

14.Heider, F. (1958). The Psychology of Interpersonal Relations. New York: Wiley.

15.Hubner, J.F., Sichman, J.S., Boissier, O., Towards a model which comprises the structural, functional, and deontic aspects of a MAS organization. In: Proc. 1st International Joint Conference on Autonomous Agents and Multi-Agent Systems (AAMAS'02), pages 501502, Bologna, Italy, July 2002.

16. Osburn, J., An overview of social role valorization theory. In The International Social Role Valorization Journal, 3(1), 7-12, 1998.

17.Kemper, Theodore D. Power, Status, and Emotions: A Sociological Contribution to A Psychophysiological Domain, In Approaches to Emotion, edited by Klaus R. Scherer, Paul Ekman. 375. Hillsdale, N.J.: Lawrence Erlbaum Associates, 1984.

18.Radcliffe-Brown, A.R., On Social Structure, In Journal of the Royal Anthropological Society of Great Britain and Ireland, Vol. 70, 1940, pp. 1-12.

19.Scott J., Social Network Analysis: A Handbook. Sage Publication, April 11, 1996.

20.Stokman F. N., Social Networks. International Encyclopedia of the Social \& Behavioral Science, Editors-in-chief, NJ Smelser \& PB Baltes, 2001.

21.Tambe, M., Scerri, P., \& Pynadath, D. V. (2002). Adjustable Autonomy: from theory to implementation. In Workshop on Autonomy Delegation and Control (Hexmoor and Falcone Eds), AAAI-2002, Edmonton, CA, AAAI Press

22.Trzebiatowski, G. L., The role concept for agents in multi-agent systems.

23.Wolfensberger, W., The principle of normalization in human services. Toronto: National Institutue on Mental Retardation, 1972.

24.Zack, Michael H., Researching Organizational Systems using Social Network Analysis. In Proceedings of the 33rd Hawaii International Conference on System Sciences, Maui, Hawaii, January, 2000. 